\title{
Author Index Vol. 69, 1996
}

The authors of the abstracts of the 11th Workshop on Surfactant Replacement are indexed separately in Vol. 69, No. 3 (p. 224), 1996.

Alfonso, L.F. 94,318 Allen, J.W. 268 Alvarez, F.J. 94,318 Alvaro,R.E. 357 Anastácio, A.S. 230 Angelopoulous, M. 140 Apricena, F. 350 A $\pi$ i,K. 307 Amaiz,A. 94,318 Asselin, C. 342

Ballèvre, L. 389 Bancalari,E. 101, 146 Basu, T.K. 133 Beaufort, A.J. de 284 Bel, F. van 284 Beratis, N.G. 76 Berger, H.M. 284 Berry, S.M. 225 Berthon, D. 51 Bessler, H. 376 Blais, S. 342 Blanco, L. 37 Bódis, J. 36 Bolande, R.P. 109 Bor, M. van de 284 Boudreau, F. 342 Broccia, M.L. 293 Brockway, P.D. 60 Brozanski, B.S. 67 Burri, P.FI. 178

Cable, S. 12 Calvert,R. 12 Carman, K. 368 Cates,D.B. 357 Chheda, S. 128 Chuai,J. 327 Colin, S. 12 Curstedt,T. 184

Daood, M. 67 Dauça, M. 12 Dauncey, M.J. 51 De Potter, W. 22 Diez-Pardo, J.A. 94

Dimenstein, R. 230 Donangelo, C.M. 230

El-Mohandes, A.E. 249

Fajardo, C.A. 357 Fando, J.L. 37 Ferrante, A. 368 Ferriero, D.M. 327 Figueroa, H. 399 Fine, N. 225 Fleit, H.B. 1,235 Flynn,J. 101 Foust, R. 275 Frank, L. 193 Frimas, C. 76 Furuhashi,Y. 307 Gall,D.G. 60 Garcia, A.M. 37 Gesink, B.J. 284 Giavini, E. 293 Godel,J.C. 133 Golde, L.M.G van 182 Goldman, A.S. 128 Goto, K. 307 Greenough, A. 197 Groneck, P. 188 Guignard, J.-P. 389

Haaland. K. 350 Haider, A.Z. 357 Halliday, H.L. 186 Hardin,J.A. 60 Harms, D. 298 Hart,J. 376 Haugvicová, R. 405 Hehre, D. 146 Herpin,P. 51 Herrera, E. 37 Heydeck,D. 383 Hibler,S. 84 Hodges, P.E. 133 Hodges, R.S. 133 Huang, J. 146

Ibe, B.O. 153

Jarvis,J.N. 225 Johansson, J. 184 Johnson-Robbins, L.A. 249 Johnston, R.B., Jr. 172 Kechner,N. 275 Keiser,J.F. 249 Keller, J.M. 12 Keuper, H. 298 Klinge,J. 298 Kobasiuk, CD. 1 Kofstad,J. 350 Kubová, H. 405 Kwiatkowski, K. 357

La Gamma, E.F. 1 Lambert, G. 140 Laroche, S. 22 LeDividich, J. 51 Leheup, B. 12 Leistikow, E.A. 109 Leitz, H. 140 Lekka, M. 119 Lloyd, J.S. 67 Lopes Cardozo, R.H. 284 Louis, T.M. 109 MacGilvray, S. 140 McPhee,A. 368 Makri,M. 76 Malinak,C. 257 Mändar,R. 30 Mares, P. 405 Martin, M.E. 37 Martin-Hidalgo, A. 37 Matsuzawa, K. 307 Ménard, D. 12 Menegola, E. 293 Mikelsaar, M. 30 Milner,A.D. 199,210 Mizutani, S. 307 Moison, R.M.W. 284 Moore, H.T. 225 413

Nakazato,H. 307 Nandoskar, M. 368 Navarro, H. 146 Ng,M.L. 133 Nilsson,G. 184 Notti, I. 376 Nowaczyk, B. 357

Obladen,M. 119 Okogbule-Wonodi, A.C. 153 Omar, S.A. 1

Pabst,H.F. 133 Palkowetz, K.H. 128 Papies,B. 383 Pascual,R. 399 Payne, N.R. 235 Peckham, C. 174 Philip, A.G.S. 175 Pinxteren, J. 22 Poulos,A. 368 Prati,M. 293

Qi,B. 94 Quero,J. 101

Raivio, K.O. 170 Raj,J.U. 84, 153 Rassin, D.K. 128 
Rauch,R. 298 Rehan,V.K. 357 Relier,J.-P. 201 Ricohi,R. 293 Ries, M. 298 Rigatto, H. 357 Robertson, B. 180, 184 Roigas,C. 383 Roigas,J. 383 Ruud Hansen, T.W. 268

Salinas, M. 37 Saugstad, O.D. 190 Scheid,A. 119 Schilt,J. 12 Segerer,H. 119 Shaffer, T.H. 275 Sheldon, R.A. 327 Shimizu,M. 243 Silverstein, F.S. 257 Simmens, S.J. 249 Sirota, L. 376 So,B.H. 243 Speer,Ch.P. 166,188 Stiris,T.A. 101 Straussberg, R. 376 Suguihara,C. 101, 146 Sulyok, E. 36

Thonney, M. 389 Thoresen, M. 350 Thorneloe, K. 342 Toga,H. 84 Tomoda,Y. 307 Tovar,J.A. 94,318 Trugo, L.C. 230 Trugo,N.M.F. 230 Tschanz, S.A. 177 Tsujimoto, M. 307

Vagenakis, A.G. 76 Valls-i-Soler, A. 94,275, 31 í Van Acker, K.J. 22 Vanderauwera, J.-C. 22 Van Reempts, P.J. 22 Varvarigou, A. 76 Volpe,J.J. 167

Wagner, M.H. 119 Wartman, F.S. Ill 109 Watanabe,T. 243 Watchko,J.F. 67 Wolfson, M.R. 275

Wouters, A. 22

Yanagisawa, M. 243 Yu,X.-Q. 146

414

Author Index Vol. 69, 1996 